\title{
Erratum to: Altered serotonin transporter availability in patients with multiple sclerosis
}

\author{
Swen Hesse • Franziska Moeller • David Petroff • Donald Lobsien • Julia Luthardt • \\ Ralf Regenthal • Georg-Alexander Becker • Marianne Patt • Eva Thomae • \\ Anita Seese • Philipp M. Meyer • Florian Then Bergh • Osama Sabri
}

Published online: 15 May 2014

(C) Springer-Verlag Berlin Heidelberg 2014

Erratum to: Eur J Nucl Med Mol Imaging (2014) 41:827-835

DOI 10.1007/s00259-013-2636-z

Owing to an oversight in typesetting, the images in Fig. 1a on page 831 incorrectly duplicated those in Fig. 1b. The correct Fig. 1a is given here.

The publisher apologises for this unfortunate error.

The online version of the original article can be found at http://dx.doi.org/ 10.1007/s00259-013-2636-z.

S. Hesse $(\bowtie) \cdot J$. Luthardt $\cdot$ G.-A. Becker $\cdot$ M. Patt $\cdot$ A. Seese $\cdot$

P. M. Meyer $\cdot$ O. Sabri

Department of Nuclear Medicine, University of Leipzig,

Liebigstraße 18, 04103 Leipzig, Germany

e-mail: swen.hesse@medizin.uni-leipzig.de

F. Moeller $\cdot$ E. Thomae $\cdot$ F. T. Bergh

Department of Neurology, University of Leipzig, Leipzig, Germany

D. Petroff

Coordinating Centre for Clinical Studies, University of Leipzig,

Leipzig, Germany

D. Lobsien

Department of Neuroradiology, University of Leipzig, Leipzig,

Germany

\section{R. Regenthal}

Clinical Pharmacology, Rudolf-Boehm-Institute of Pharmacology

and Toxicology, University of Leipzig, Härtelstraße 16-18,

04107 Leipzig, Germany

\section{S. Hesse $\cdot$ O. Sabri}

Integrated Research and Treatment Center (IFB) Adiposity Diseases,

University of Leipzig, Liebigstraße 21, 04103 Leipzig, Germany 


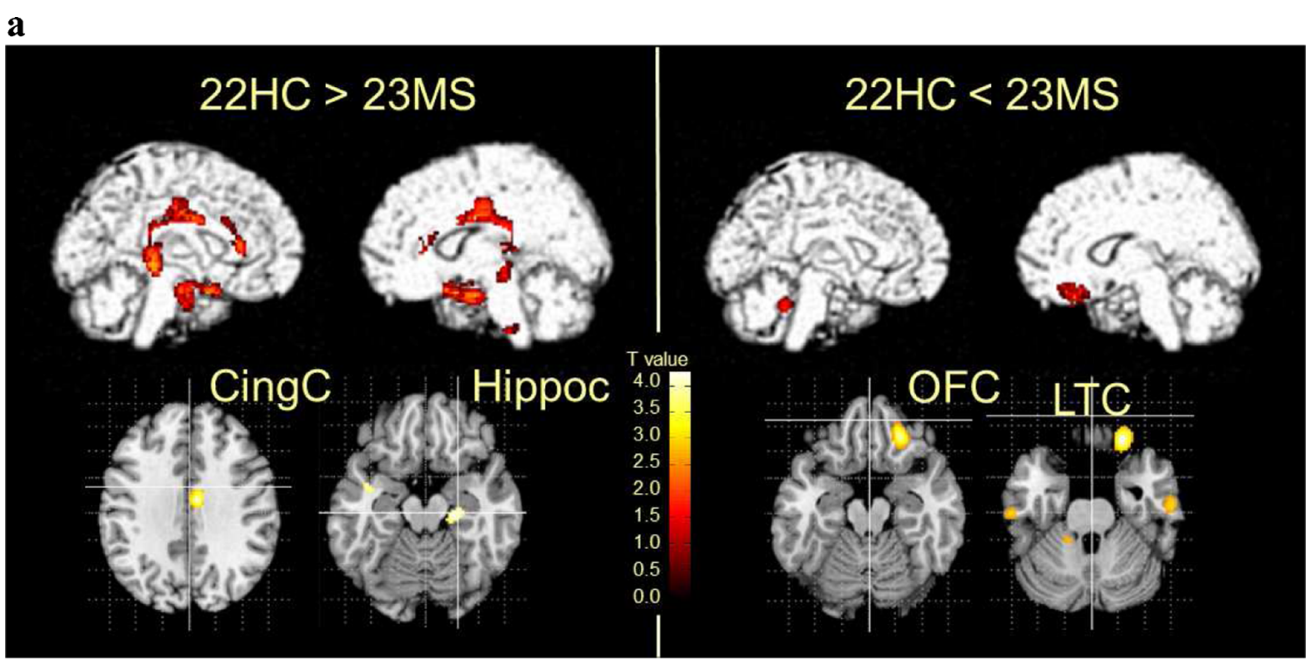

\begin{tabular}{|c|c|c|}
\hline $\begin{array}{c}\text { Talairach coordinates } \\
\qquad(x y z)\end{array}$ & T value & Brain region \\
\hline \multicolumn{3}{|c|}{$22 \mathrm{HC}>23 \mathrm{MS}, \mathrm{p} \leq 0.005$, min. $30 \mathrm{voxe} / /$ cluster } \\
\hline $\begin{array}{lll}6 & -14 & 36\end{array}$ & 4.33 & Cingular Cortex R \\
\hline $18-826$ & 3.89 & ParahippocampalArea $\mathrm{R}$ \\
\hline $\begin{array}{lll}22 & -26 & 10\end{array}$ & 3.43 & Hippocampus R \\
\hline$-44-4-16$ & 3.15 & Temporal Cortex $L$ \\
\hline $\begin{array}{lll}-6 & 30 & 12\end{array}$ & 3.11 & Anterior Cingular Cortex $\mathrm{L}$ \\
\hline \multicolumn{3}{|c|}{$22 \mathrm{HC}<23 \mathrm{MS}, \mathrm{p} \leq 0.005$, min. 30 voxel $/$ cluster } \\
\hline $2432-18$ & 4.80 & Orbitofrontal Cortex R \\
\hline $60-16-30$ & 4.61 & Lateral Temporal Cortex $\mathrm{R}$ \\
\hline$-58-24-24$ & 3.35 & Lateral Temporal Cortex L \\
\hline
\end{tabular}

